\title{
Inhibition of return is no hallmark of exogenous capture by unconscious cues
}

\author{
Isabella Fuchs ${ }^{1 *}$ and Ulrich Ansorge ${ }^{1,2}$ \\ Faculty of Psychology, University of Vienna, Vienna, Austria \\ 2 Institute of Cognitive Science, University of Osnabrück, Osnabrück, Germany
}

\author{
Edited by: \\ Nicola De Pisapia, University of \\ Trento, Italy \\ Reviewed by: \\ David Souto, University College \\ London, UK \\ Xiaolin Zhou, Peking University, \\ China \\ *Correspondence: \\ Isabella Fuchs, Faculty of \\ Psychology, University of Vienna, \\ Liebiggasse 5, A-1010 Wien, Vienna, \\ Austria. \\ e-mail: isabella.fuchs@univie.ac.at
}

\begin{abstract}
Inhibition of irrelevant information and response tendencies is a central characteristic of conscious control and executive functions. However, recent theories in vision considered Inhibition of Return (IOR: slower responses to attended than unattended positions) to be a hallmark of automatic exogenous capture of visual attention by unconscious cues. In the present study, we show that an unconscious cue that exogenously captures attention does not lead to IOR. First of all, subliminal cues with a contrast different from a searched-for target contrast capture attention independently of their match of contrast polarity to the search criteria. This is found with a short cue-target interval (Exp. 1). However, the same cues do not lead to IOR with a long cue-target interval. The lack of IOR is also verified for several intermediate intervals (Exp. 2), for high-contrast cues and low-contrast targets (Exp. 3), and with lower luminance cues presented on a CRT screen (Exp. 4). Finally, no capture effect but IOR is found for consciously perceived anti-predictive cues (Exp. 5). Together the results support the notion of a double dissociation between IOR and exogenous capture and are in line with a decisive role of consciousness for inhibition.
\end{abstract}

Keywords: vision, attention, subliminal, cueing, inhibition of return, attentional capture, exogenous capture

\section{INTRODUCTION}

Conscious control allows inhibition of irrelevant information (cf. Baars, 2002; Botvinick et al., 2004), but inhibition has also been found with task-relevant unconscious stimuli (Lau and Passingham, 1998). Recently, however, Mulckhuyse and Theeuwes (2010) argued that Inhibition of Return (IOR) could be a hallmark of exogenous capture of visual attention by unconscious cues. In this context, exogenous capture denotes attentional capture by a truly task-irrelevant stimulus, and IOR denotes that participants respond slower to recently attended but now ignored positions than to less attended positions (cf. Taylor and Klein, 1998). For example, presenting a cue with a cue-target Stimulus Onset Asynchrony (SOA) of less than about $300 \mathrm{~ms}$ at one of two possible target positions, attentional capture to the cue is reflected in facilitated responses to a target at the same position (SP) as the cue. This facilitation is found relative to the responses to a target at a different position (DP) than the cue. However, with SOAs of more than about $300 \mathrm{~ms}$, this cueing effect reverses into IOR and responses will often be slower in SP than DP conditions.

Mulckhuyse and Theeuwes (2010) argued that exogenous capture and IOR by unconscious cues are flip sides of one shared process of initially activating and subsequently inhibiting one cued location representation within the retinotopic map of the Superior Colliculi, a midbrain structure that is fed by the visual input of the magno-cellular projection from the retinal ganglion cells. Support for this supposition comes from the observations that the Superior Colliculi indeed seem to play an active role (I) in exogenous capture (cf. Rafal et al., 1991) and (II) in unconscious capture (cf. Ansorge, 2003; Fuchs and Ansorge, 2012) as well as (III) in IOR (cf. Dorris et al., 2002).
However, it is also possible that exogenous capture and IOR are brought about by dissociable cortical structures rather than by one shared sub-cortical structure (e.g., Lupiánez, 2010; Hu and Samuel, 2011). For example, exogenous capture (e.g., Itti et al., 1998) and unconscious capture (e.g., Zhaoping, 2008) could also rely on processing of visual input in early visual cortex (e.g., V1) whereas IOR could be brought about by a different cortical area, for example, posterior parietal cortex (e.g., Mayer et al., 2004; Toffanin et al., 2011). According to this line of reasoning, it might well be that exogenous unconscious capture and IOR are less tightly coupled to one another than assumed so that at least in some instances exogenous unconscious capture could be observed without subsequent IOR. This is of some relevance. If it can be shown that unconscious exogenous capture and IOR are not flip sides of one another, one could not rely on one of the effects, for example, IOR, to infer with certainty that the other effect, for example, exogenous capture, has also taken place. This, however, is exactly the conclusion that has been drawn by Mulckhuyse and Theeuwes (2010).

In addition, research with clearly visible cues has shown that IOR is not only a consequence of exogenous attentional capture. To reiterate, exogenous capture can only be demonstrated when the cue is truly irrelevant and does not fit to the top-down control settings of the participants. However, IOR can also be found after top-down contingent capture. For example, having their participants search for either abrupt onset targets or color targets, Gibson and Amelio (2000) demonstrated that abrupt onset cues only captured attention and led to subsequent IOR if the participants searched for abrupt onset targets. Capture and IOR, however, were absent when an abrupt onset cue was used 
but targets were defined by colors. Gibson and Amelio (2000) concluded that both, capture and IOR depended on a match of the abrupt onset cues to the top-down control settings of their participants which were set up to search for the targets (cf. Folk et al., 1992; see also Pratt et al., 2001; Prinzmetal et al., 2011).

This was the point of departure for the present study. We wanted to test whether IOR is indeed a necessary consequence of exogenous unconscious capture. Exogenous capture would only be demonstrated where two conditions are fulfilled: visual cues would have to (I) not match the participants' top-down search templates for relevant features and (II) be uninformative about target positions (cf. Folk et al., 1992). Because criterion (I) must not be met to find IOR (e.g., Gibson and Amelio, 2000) and because Mulckhuyse et al. (2007) only used cues of a task-relevant contrast polarity, it is possible that unconscious IOR reflected top-down contingent capture based on a fit of the cue's contrast polarity to the search settings rather than (a consequence of) exogenous capture by a truly irrelevant cue. Therefore, it still needs to be tested whether IOR is or is not a hallmark of unconscious exogenous attentional capture.

In five Experiments, our participants reported the presence of targets in unconsciously cued SP and DP conditions. Attentional capture was verified as faster target detection in SP than DP conditions with short SOAs (cf. McCormick, 1997). IOR was tested as slower target detection in SP than DP conditions with long cue-target SOAs. Crucially, we also varied whether or not the cues matched the top-down search sets for contrast polarity. Our cues either had the same contrast polarity as the searched-for targets, or the cues had the opposite contrast polarity to the searchedfor targets. Thus, only the same- but not the opposite-polarity cues matched the participants' top-down search templates for target contrasts. For example, if participants searched for dark targets, dark cues were top-down matching but light cues were non-matching. If it is true that IOR is a hallmark of unconscious exogenous capture, we expected IOR in the non-matching conditions with an opposite-polarity cue because it has been shown that truly exogenous capture does not depend on a match of the contrast polarity sign (Steinman et al., 1997). In the final Experiment 5 we also tested attentional effects of anti-predictive consciously and unconsciously perceived cues (cf. McCormick, 1997). If consciousness has a supportive role for inhibition, we expected to find IOR with conscious cues even if it was lacking with unconscious cues.

\section{EXPERIMENTAL SECTION \\ EXPERIMENT 1 \\ Methods}

In Experiment 1 (with a TFT monitor) eight participants searched for black $\left(23 \mathrm{~cd} / \mathrm{m}^{2}\right)$ and another eight for white $\left(122 \mathrm{~cd} / \mathrm{m}^{2}\right)$ targets against a gray background $\left(72.5 \mathrm{~cd} / \mathrm{m}^{2}\right.$, Weber contrast for both targets was $\left.c_{w}= \pm 0.68\right)$. Figure 1A depicts sample trials: the cue (a ring of $3.0^{\circ} \times 3.0^{\circ}$ size and of a strength of $0.25^{\circ}$ ) was presented left or right on the screen (with an eccentricity of $6.7^{\circ}$ ) $16 \mathrm{~ms}$ before two placeholder rings (of the same size) appeared: one in the middle and the other one on the opposite side of the screen. The target was a disk (of $1.9^{\circ} \times 1.9^{\circ}$ size) and

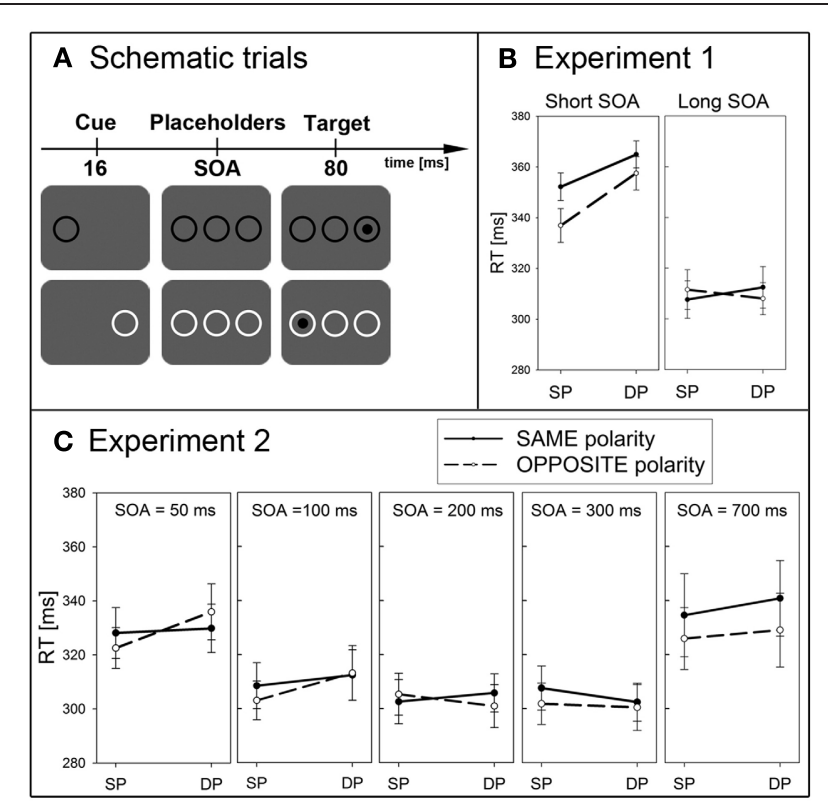

FIGURE 1 | (A) Depicted are schematic trials. The top row shows a dark target on the right side, cued by a dark cue at the left side (same-polarity and DP condition). The bottom row also depicts a dark target, but preceded by a light cue and again on the different position (opposite-polarity and DP condition). (B) Depicted are mean RTs (between 280 and 380 ms) and standard errors of the mean (error bars) of all participants, plotted separately for the short (left panel) and long SOA (right panel) of Experiment 1. Results are shown for SP (left side of each panel) and DP conditions (right side of each panel), and plotted separately for same- (solid lines) and opposite-polarity cues (dashed lines). (C) Depicted are mean RTs (between 280 and $380 \mathrm{~ms}$ ) and standard errors of the mean (error bars) of all participants, plotted separately for the five different SOAs (separate panels, from left to right for $\mathrm{SOA}=50,100,200,300$, and $700 \mathrm{~ms}$ ) of Experiment 2.

could appear either centered in the left or right ring. In the short SOA condition, the target appeared along with the placeholders. In the long SOA condition, the target was shown $1 \mathrm{~s}$ after the onset of the two placeholder rings. The target was shown in $80 \%$ of the trials. The cue and the targets were equally likely left or right, and the target was equally often at the cued position (SP condition) or opposite of the cue (DP condition). The cue was, therefore, uninformative with respect to the target position and the cue was also invisible (or hard to see) because of strong flicker fusion with the placeholder onsets during the tiny interval between the cue and the two other placeholder rings. For every participant a same- or an opposite-polarity cue (with luminance values as for the respective targets) equally likely preceded the target. Different conditions were presented in a randomized sequence of 200 trials. To assess capture (in the short SOA) and IOR (in the long SOA), participants reported the target's presence by a button press. After the target-detection task, participants were informed that one of the rings (i.e., the cue) appeared one frame earlier than the others, and instructed to report the cue position. Participants pressed the left button if they saw the cue on the left and the right button if they saw the cue on the right. In this task, the participants' unconsciousness about the cues would 
be reflected by a small $d^{\prime}$ value, with $d^{\prime}=0$ indicating chance performance.

\section{Results}

Participants performed very well on the target-detection task (mean $d^{\prime}$ of target detection $=4.3$ ). Only target-present trials were included in the analyses, and trials with incorrect responses (i.e., misses; 1.1\%) and outliers [reaction times (RTs) deviating more than two standard deviations from the mean; $4.0 \%$ ] were excluded. See also Figure 1B for the RT results. A repeatedmeasurements ANOVA of the mean correct RTs with the variables cue position (SP vs. DP), cue contrast polarity (same vs. opposite), and SOA (short vs. long) led to the following results. Responses to targets preceded by an SP cue $(327 \mathrm{~ms})$ were generally faster than in DP conditions $(336 \mathrm{~ms})$, as indicated by a main effect of cue position, $F(1,15)=13.6, p<0.01, \eta_{p}^{2}=$ 0.48 . Participants also responded faster in the long SOA $(\mathrm{RT}=$ $310 \mathrm{~ms}$ ) than the short SOA (RT $=353 \mathrm{~ms})$, as indicated by a significant main effect of SOA: $F(1,15)=101.8, p<0.001, \eta_{\mathrm{p}}^{2}=$ 0.87 , and in opposite- $(\mathrm{RT}=328 \mathrm{~ms})$ than same-polarity $(\mathrm{RT}=$ $334 \mathrm{~ms}$ ) conditions, reflected in a main effect of contrast polarity, $F(1,15)=8.5, p<0.05, \eta_{\mathrm{p}}^{2}=0.36$. Significant two-way interactions between cue polarity and SOA, $F(1,15)=5.2, p<0.05$, $\eta_{\mathrm{p}}^{2}=0.26$, and between cue position and SOA $F(1,15)=22.7$, $p<0.001, \eta_{\mathrm{p}}^{2}=0.60$, prompted post-hoc Bonferroni-adjusted $t$-tests of cueing effects (SP vs. DP) split up for each combination of SOA and contrast polarity. Importantly, these tests revealed a significant cueing effect in the short SOA for both, same-polarity (SP: RT $=352 \mathrm{~ms}$, DP: RT $=365 \mathrm{~ms}, p<0.01$ ) and oppositepolarity conditions (SP: RT $=337 \mathrm{~ms}$, DP: $\mathrm{RT}=358 \mathrm{~ms}, p<$ 0.01 ). In the long SOA, no significant effect was found, neither for same-polarity (SP: RT $=308 \mathrm{~ms}$, DP: $\mathrm{RT}=312 \mathrm{~ms}, p=0.19$ ) nor opposite-polarity conditions (SP: RT $=312 \mathrm{~ms}$, DP: RT $=$ $308 \mathrm{~ms}, p=0.50$ ).

After finishing the target-detection task, participants were asked whether they had been aware of the cue (i.e., one of the rings starting earlier) during the target-detection task. All participants reported a subjective unawareness of the cues during the target-detection task. However, as an objective measure of cue awareness, we next ran a cue-discrimination task, and $d^{\prime}$ for cue detection was calculated from this task. To be precise, in the cue-discrimination task, correct reports of cues on the right counted as hits, and incorrect reports of cues on the right as false alarms, and $d^{\prime}$ was calculated as the difference between the $z$-transformed probabilities of the hits minus the $z$-transformed probabilities of the false alarms. $d^{\prime}$ will be zero in the case of chance performance (or invisibility of the cues) and can infinitely increase with an ever increasing discrimination performance. On average, cue detection was above chance level [mean $d^{\prime}=0.24$, $t$-test against zero, $t(15)=2.5, p=0.024]$. However, restricting our analysis to only the worst cue discriminators among the participants, the participants remained completely unaware of the cue [mean $\left.d^{\prime}=-0.02, t(7)=-0.55, p=0.60\right]$, and yet the RT effects reported above could be replicated, with significant main effects for cue position and SOA, and a significant interaction between these variables reflecting that there was a cueing effect in the short SOA $(p<0.01)$ but no IOR in the long SOA $(p=0.27)$.

\section{Discussion}

We found cueing effects in the short SOA for both, sameand opposite-polarity conditions. This is evidence for exogenous capture because only in the same- but not in the oppositepolarity conditions, the cues would have matched the top-down search template for target contrasts. In addition, exogenous capture was also found with the participants that remained unaware of the cues. This demonstrated exogenous unconscious capture. Crucially, however, we could not find any evidence of IOR in the long SOAs. This finding is at variance with the claim that IOR is a hallmark of unconscious exogenous capture. Before we could conclude this with certainty, additional possible explanations for the lack of IOR had to be tested. First, the long SOA of $1 \mathrm{~s}$ could have been an unfortunate choice, if IOR occurred earlier and had vanished after $1 \mathrm{~s}$. To test this possibility, we conducted Experiment 2, where we used five intermediate SOAs between 50 and $700 \mathrm{~ms}$. Second, the luminance values of our stimuli and background were different to previous studies that reported IOR (cf. Mulckhuyse et al., 2007). This might be crucial because lower target contrasts can lead to larger IOR compared to higher contrasts (e.g., Hunt and Kingstone, 2003). This was tested in Experiment 3. Third, the current Experiment differed in two further respects from the study by Mulckhuyse et al. (2007): the use of a CRT screen and the cues' luminance values. These changes were addressed in Experiment 4.

\section{EXPERIMENT 2 Methods}

With 18 new participants, we used the same experimental set up as in Experiment 1, except for the SOAs that were 50, 100, 200, 300 , or $700 \mathrm{~ms}$, presented in a random order.

\section{Results and discussion}

Participants performed very well on the target-detection task (mean $d^{\prime}=4.2$ ). Again, only target-present trials were analyzed, and trials with incorrect responses $(2.1 \%)$ and outlying RTs (further 2.3\%) were excluded. For the RT results see Figure 1C. A repeated-measurements ANOVA of the mean correct RTs with the variables cue position (SP vs. DP), cue contrast polarity (same vs. opposite), and $S O A$ (five steps) led to a significant main effect of SOA, $F(4,68)=11.2, p<0.001, \eta_{p}^{2}=0.40$ (for SOA of $50 \mathrm{~ms}$ : $\mathrm{RT}=329 \mathrm{~ms}$, SOA of $100 \mathrm{~ms}: \mathrm{RT}=309 \mathrm{~ms}$, SOA of $200 \mathrm{~ms}: \mathrm{RT}=$ $304 \mathrm{~ms}$, SOA of $300 \mathrm{~ms}$ : RT $=303 \mathrm{~ms}$, and SOA of $700 \mathrm{~ms}: \mathrm{RT}=$ $333 \mathrm{~ms}$ ). All other tests failed to reach significance.

All participants reported a subjective unawareness of the cues during the target-detection task, but the cue detection-block revealed above chance accuracy for the shortest and longest SOA (mean $d^{\prime} s=0.35$ and 0.44 respectively, $p<0.05$ ), whereas the cue remained below the objective threshold in the other SOAs (all $p s>0.05$ ).

\section{Discussion}

In Experiment 2 we used the exact same procedure as in Experiment 1 except for the SOAs. Although the cues initially captured attention (at a very short SOA of Exp. 1), the capture effect seems to wear off very rapidly, as indicated by non-significant 
effects for all SOAs in Experiment 2. This result also rules out the possibility that IOR occurred at an earlier interval between 50 and $700 \mathrm{~ms}$.

\section{EXPERIMENT 3}

In Experiment 3, we tested whether IOR depended on contrast strength. In past research it was found that lower target contrasts can lead to larger IOR compared to higher target contrasts (e.g., Hunt and Kingstone, 2003). For our tests, we used high-contrast cues preceding low-contrast targets at four different SOAs.

\section{Methods}

With 14 new participants, we used a similar experimental set up as in Experiment 1. Here, all participants searched for a lowcontrast target $\left(10.5 \mathrm{~cd} / \mathrm{m}^{2}\right.$, Weber contrast $\left.c_{w}=0.62\right)$ preceded by a high-contrast cue $\left(104 \mathrm{~cd} / \mathrm{m}^{2}\right.$, Weber contrast $\left.c_{w}=15.0\right)$ against a black background $\left(6.5 \mathrm{~cd} / \mathrm{m}^{2}\right)$. The reported luminance values match those used in the study by Hunt and Kingstone (2003). Furthermore, we also varied the SOAs: In half of the trials, the target again appeared simultaneously with the two other placeholders, whereas in the other half of the trials the target followed equally often after an SOA of $800,1,000$, or 1,200 ms. The additional long SOAs of 800 and 1,200 ms were chosen corresponding to the study by Hunt and Kingstone (2003; where the interval between cue and target onset was 1,160 ms, and between cue offset and target onset $860 \mathrm{~ms}$ ). The target again appeared equally often at the same (SP) or different position (DP) as the cue. Conditions were presented in a randomized sequence of 240 trials including 20\% catch trials. Cue visibility was assessed in a second block consisting of 60 trials.

\section{Results}

Participants performed well in the target-detection task even though the target was difficult to see (mean $d^{\prime}=2.1$ ). Again, only target-present trials were analyzed, and trials with incorrect responses $(20.7 \%)$ and outlying RTs (4.4\%) were excluded. See Figure 2A for the RT results. First, we tested for IOR in

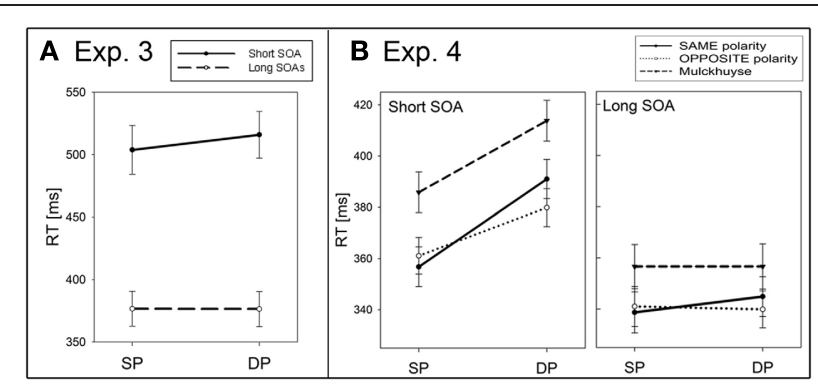

FIGURE 2 | (A) Depicted are the results (mean RTs between 350 and $550 \mathrm{~ms}$ ) of Experiment 3. Mean RT was faster if the cue was at the same position (SP) as the target than if the cue was at a different position (DP) than the target. This was found with a short cue-target stimulus onset asynchrony (SOA; solid line) but not with a long SOA (dashed line). (B) Analogous results (mean RTs between 325 and 425 ms) of Experiment 4 are plotted separately for the short (solid line) and long SOAs (dashed line), for three different conditions separately (see figure legend). For further details refer to the Methods sections of Experiments 3 and 4. only the long SOAs, and calculated a repeated-measurements ANOVA with the variables cue position (SP vs. DP), and SOA $(800,1,000$, or $1,200 \mathrm{~ms})$ on RTs. There was neither a significant main effect of SOA nor cue position, nor a significant interaction between these variables (all $p$ s $>0.18$ ). Therefore, we collapsed across different long SOAs for a subsequent ANOVA, with the variables cue position (SP vs. DP), and SOA (short vs. long) on RTs. Again we found a significant main effect for SOA, $F(1,13)=63.1, p<0.001, \eta_{\mathrm{p}}^{2}=0.83$, indicating faster responses in the long SOAs $(\mathrm{RT}=377 \mathrm{~ms})$ than in the short SOA (RT = $510 \mathrm{~ms})$. A significant interaction between cue position and SOA, $F(1,13)=7.7, p<0.05, \eta_{p}^{2}=0.37$, and post-hoc Bonferroniadjusted $t$-tests revealed a significant cueing effect in the short SOA (SP: RT $=504 \mathrm{~ms}$, DP: RT $=516 \mathrm{~ms}, p<0.05$ ) and no effect in the long SOAs (SP: RT $=377 \mathrm{~ms}$, DP: RT $=376 \mathrm{~ms}$, $p=0.94)$.

Importantly, again, all participants reported a subjective unawareness of the cues during the target-detection task. Objectively, participants performed above chance level in the cuedetection task (mean $d^{\prime}=1.65, p<0.001$ ), and performance did not differ across SOAs, $F(3,39)<1$.

\section{Discussion}

When presenting a low-contrast target cued by a high-contrast cue, we failed to find IOR in the long SOAs, although we found a cueing effect in the short SOA. This finding rules out that the lack of IOR in the present Experiment 1 could be explained by the high contrast strength of the target relative to the background, which facilitated target perception and undermined the attentional effect of the cues.

The results of Experiment 3 also prompted a question: because target- and cue-detection were both objectively, and almost equally bad, does this mean that the participants were unaware of the low-contrast targets? Not necessarily. During the targetdetection task, participants actively searched for the targets. Although active top-down search for a stimulus does not always lead to stimulus awareness (e.g., Ansorge et al., 2009), a wealth of research suggests that active search for a stimulus has the potential to increase stimulus awareness (cf. Mack and Rock, 1998; Simons, 2000). Therefore, our participants probably saw the targets but failed to subjectively see the cues during target-detection because they actively searched for only the targets. By the same token, the participants probably became increasingly aware of the cues during cue-detection, when they actively searched for the cues. In line with this, participants indeed occasionally reported becoming also subjectively aware of the cues during the cuedetection task. In conclusion, we can understand that during target-detection the participants were aware of the targets and at the same time unaware of the cues, if we take into account the awareness-mediating role of target-directed vs. cue-directed active search.

\section{EXPERIMENT 4}

Experiment 4 was conducted on a CRT screen and participants searched for three different target contrasts in three separate blocks: black targets, white targets, and targets matching the luminance conditions reported in Mulckhuyse et al. (2007). 


\section{Methods}

Experiment 4 used the same procedures as Experiment 1, except for the following changes: A CRT monitor (as in Mulckhuyse et al., 2007) was used instead of a TFT screen. All participants $(N=18)$ searched for three different target luminances in three separate blocks (with different block orders balanced across participants): one block with black $\left(20 \mathrm{~cd} / \mathrm{m}^{2}\right)$ targets against a gray background $\left(55 \mathrm{~cd} / \mathrm{m}^{2}\right.$; Weber contrast $\left.c_{w}=-0.8\right)$, one with white $\left(90 \mathrm{~cd} / \mathrm{m}^{2}\right)$ targets against the same gray background $\left(c_{w}=\right.$ +0.8 ) - in these blocks, a cue of either the same or the opposite contrast polarity preceded the target, - and a third block with targets and backgrounds with the same luminance values as reported in Mulckhuyse et al. [i.e., a target in dark-gray $\left(13 \mathrm{~cd} / \mathrm{m}^{2}\right)$ against a black background $\left.\left(5 \mathrm{~cd} / \mathrm{m}^{2}\right), c_{w}=1.6\right]$. The latter condition will henceforth be referred to as the "Mulckhuyse condition." In this condition, only cues matching the target luminance were used. In all blocks, the target was either presented along with the other placeholders $(\mathrm{SOA}=0 \mathrm{~ms}$ ) or with an SOA of $1 \mathrm{~s}$. Again, participants searched for the targets in a random sequence of all conditions within each block of 200 trials.

\section{Results}

Participants performed very well on the target-detection task (mean $d^{\prime}=4.7$ ). Again, only target-present trials were analyzed, and trials with incorrect responses (1.1\%) and outlying RTs $(2.7 \%)$ were excluded. See Figure 2B for the RT results. A repeated measurements ANOVA of the mean correct RTs with the variables cue position (SP vs. DP), cue condition (same-polarity vs. opposite-polarity vs. Mulckhuyse condition), and SOA (short vs. long) led to a significant main effect for SOA, $F(1,17)=54.0, p<$ $0.001, \eta_{\mathrm{p}}^{2}=0.76$, with shorter RTs in the long $(\mathrm{RT}=346 \mathrm{~ms})$ than in the short SOA (RT $=381 \mathrm{~ms})$. Furthermore, responses in SP conditions ( $\mathrm{RT}=357 \mathrm{~ms}$ ) were generally faster than in DP conditions $(\mathrm{RT}=371 \mathrm{~ms})$, as indicated by a significant main effect of cue position, $F(1,17)=185.1, p<0.001, \eta_{\mathrm{p}}^{2}=0.92$. Also, responses in the Mulckhuyse condition were generally slower $(\mathrm{RT}=378 \mathrm{~ms})$ compared to the same- $(\mathrm{RT}=358 \mathrm{~ms}, p<0.001)$ and opposite-polarity condition ( $\mathrm{RT}=355 \mathrm{~ms}, p<0.001)$, indicated by a main effect for cue condition, $F(2,34)=46.2, p<$ $0.001, \eta_{\mathrm{p}}^{2}=0.73$. We found significant interactions between cue condition and cue position, $F(2,34)=5.4, p<0.05, \eta_{\mathrm{p}}^{2}=0.24$, between cue condition and SOA, $F(2,34)=7.1, p<0.01, \eta_{\mathrm{p}}^{2}=$ 0.30 , and between cue position and SOA, $F(1,17)=82.4, p<$ $0.001, \eta_{\mathrm{p}}^{2}=0.83$. Post-hoc Bonferroni adjusted $t$-tests revealed significant cueing effects (i.e., faster responses to SP than DP cued targets) for all cue conditions in the short SOA (RT differences, i.e., RTs to DP cues - SP cues: for same-polarity $=34 \mathrm{~ms}$, for opposite-polarity $=19 \mathrm{~ms}$, and for the Mulckhuyse condition = $28 \mathrm{~ms}$, all ps $<0.001)$. No significant results were found for the long SOA at all (RT differences, i.e., RTs to SP cues - DP cues: for same-polarity $=-6 \mathrm{~ms}$, for opposite-polarity $=1 \mathrm{~ms}$, and for the Mulckhuyse condition $=0 \mathrm{~ms}$, all $p s>0.09$ ), indicating a lack of IOR in all conditions.

Importantly, again, all participants reported a subjective unawareness of the cues during the target-detection task. Also, although objective cue detection was worse in the Mulckhuyse condition (mean $d^{\prime}=1.32$ ) than in the same-polarity (mean $\left.d^{\prime}=1.98 ; p<0.05\right)$ and the opposite-polarity condition (mean $\left.d^{\prime}=2.21 ; p<0.01\right)$, participants performed above chance level in all conditions (all $p s>0.001$ ).

\section{Discussion}

Experiment 4 showed that capture can be found even with cues that are subjectively not seen but that IOR is not observed under these conditions. These findings rule out that the CRT screen or the different luminance values of the cues and background used in the study by Mulckhuyse et al. (2007) as compared to the present Experiments 1-3 accounted for the absence of IOR in the current study. Experiment 4 is a failure to replicate IOR under the conditions of Mulckhuyse et al. (2007).

\section{EXPERIMENT 5}

In Experiment 5, we tested whether IOR occurs for anti-predictive supraliminal cues. This manipulation allows for a strategic use of the cues (cf. McCormick, 1997; Ansorge, Kiss et al., 2011). Since the target can be found at the position opposite to the cue (i.e., in the DP conditions) in the majority of the trials, we expected faster responses to DP than SP conditions, when the cues were visible, but not for invisible cues. In the Introduction, we argued for a critical role of consciousness during inhibition. Therefore, in two separate blocks the cues were either rendered visible or invisible, and they appeared at the position opposite to the target on the majority of trials. We expected a qualitative difference. Based on the supportive role of consciousness for inhibition IOR was expected in supraliminal cueing conditions. Based on the lack of IOR in the preceding experiments of the present study, however, no IOR was expected in the subliminal cueing conditions.

\section{Methods}

With 16 new participants, we used a similar experimental set up as in Experiment 1, except for the following changes. First, in two separate blocks, the cues were either invisible (as in the preceding experiments) or visible. Cue visibility was achieved in one block by omitting the two additional placeholder rings (see Figure 3A). Block sequence was balanced across participants. Second, targets were preceded by DP cues in $75 \%$ and by SP cues in only $25 \%$ of the target-present trials. In this manner, IOR was encouraged.

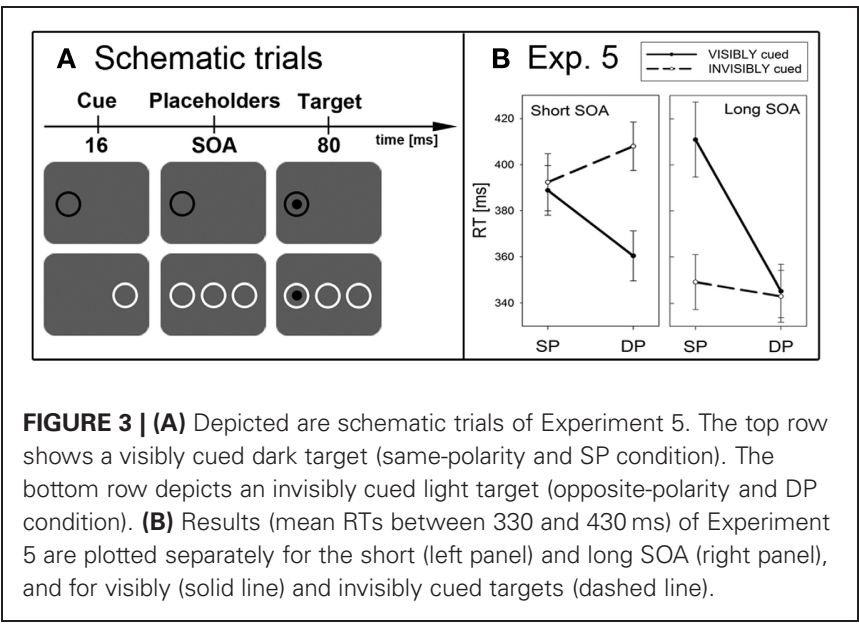


Different conditions were presented in a randomized sequence of 240 trials including 20\% catch trials. Cue awareness was assessed in a final block consisting of 80 trials.

\section{Results}

Participants performed very well on the target-discrimination task (mean $d^{\prime}=4.1$ ). Again, only target-present trials were analyzed, and trials with incorrect responses (1.9\%) and outlying RTs $(2.8 \%)$ were excluded. See Figure 3B for the RT results. A repeated-measurements ANOVA with the variables cue position (SP vs. DP), cue contrast polarity (same vs. different), cue visibility (visible vs. invisible), and SOA (short vs. long) on mean correct RTs led to the following results. Responses in SP conditions $(\mathrm{RT}=385 \mathrm{~ms}$ ) were generally slower than in DP conditions $(\mathrm{RT}=364 \mathrm{~ms})$, as indicated by a significant main effect of cue position, $F(1,15)=41.7, p<0.001, \eta_{\mathrm{p}}^{2}=0.74$. Furthermore, participants generally responded faster in the long SOA (RT = $362 \mathrm{~ms}$ ) compared to the short SOA ( $\mathrm{RT}=387 \mathrm{~ms}$ ), as indicated by a significant main effect for SOA, $F(1,15)=15.5, p<$ $0.01, \eta_{\mathrm{p}}^{2}=0.51$. We found four significant two-way interactions: between cue position and contrast polarity, $F(1,15)=5.9, p<$ $0.05, \eta_{\mathrm{p}}^{2}=0.28$, between cue position and awareness, $F(1,5)=$ 46.1, $p<0.001, \eta_{\mathrm{p}}^{2}=0.76$, between cue position and SOA, $F(1,15)=22.2, p<0.001, \eta_{\mathrm{p}}^{2}=0.60$, and between awareness and SOA, $F(1,15)=49.9, p<0.001, \eta_{\mathrm{p}}^{2}=0.77$. Importantly, post-hoc Bonferroni-adjusted $t$-tests revealed a cueing effect (SP: $\mathrm{RT}=392 \mathrm{~ms}$, DP: RT $=408 \mathrm{~ms}, p<0.05)$ in the invisible condition for the short SOA, whereas participants responded slower to $\mathrm{SP}(\mathrm{RT}=389 \mathrm{~ms})$ compared to DP cues $(\mathrm{RT}=360 \mathrm{~ms}, p<0.01)$ when the cue was visible. In the long SOA, we again found no significant effect for invisible cues (SP: RT $=349 \mathrm{~ms}$, DP: RT $=$ $343 \mathrm{~ms}, p=0.36$ ), and IOR for visible cues (SP: RT $=411 \mathrm{~ms}$, DP: $\mathrm{RT}=345 \mathrm{~ms}, p<0.001)$.

Cue detection was worse for invisible cues (mean $d^{\prime}=1.92$ ) than for visible cues (mean $d^{\prime}=3.51 ; p<0.01$ ), but participants performed above chance level in both conditions (both ps $>0.001)$.

\section{Discussion}

The results of Experiment 5 show a double dissociation between cueing effects and IOR: for anti-predictive visible cues IOR but no capture was found, whereas for unconscious or invisible cues a capture effect but no IOR was obtained (cf. McCormick, 1997). This emphasizes that IOR and exogenous capture are based on two separate mechanisms, mediated by conscious control or being more independent of control, respectively. With the visible cues, IOR was even found with the short SOA, which is in line with prior findings (cf. Tassinari and Berlucchi, 1993). One caveat of the present experiment is that the visual stimulation in the visible and invisible condition cannot be directly compared. For instance, the placeholders could have undermined capture and IOR only in the unconscious cueing conditions. Evidently, this was not the case regarding capture because capture was only found in the invisible conditions with the additional placeholders. However, future research should be devoted to understand the confounded roles of invisibility and placeholders on the lack of IOR, too.
A second important aspect of the results of Experiment 5 is that the subliminal cues used throughout this study were truly subliminal because if participants would have been aware of the cues, their strategic use for inhibition would have led to faster responses in DP than SP conditions in the subliminal condition, too.

\section{GENERAL DISCUSSION}

We found attentional capture by unconscious cues. This was reflected in faster target detection in SP than DP conditions in the short cue-target interval for both, same- and opposite-polarity conditions (Exp. 1), and these effects did not differ for very lowcontrast targets (Exp. 3) or varying luminance values of cues or targets (Exp. 4). In contrast to some previous research (cf. Ansorge et al., 2010), we observed subliminal attentional capture that was independent of the match of the cue to the searched-for target features (here: target contrast polarities). Thus, exogenous capture by the cues was ensured at least in the opposite-polarity conditions. In the current study we found an independence of the cueing effect from the match between the contrast polarity of the unconscious cue and the participants' search set.

Crucially, however, and in line with our opinion that IOR is not a hallmark of unconscious exogenous capture, we did not find IOR with longer cue-target SOAs (see also McCormick, 1997; Scharlau et al., 2006). This was found even when different intermediate SOAs were used (Exp. 2). Therefore, the lack of IOR was very likely not due to an unfortunate choice of SOAs. In addition, because we found exogenous capture with the same cues under short SOA conditions (in Exp. 1), the lack of IOR cannot be ascribed to an absence of attentional capture. Finally, whether or not the cues were relevant did not change this major result. Crucially, IOR but no capture was found in a control condition with consciously perceived cues (Exp. 5). Together, these results support the conclusion that capture and IOR do not necessarily rely on the same mechanism (cf. Prinzmetal et al., 2011), and must not be brought about by one shared two-phase process in the Superior Colliculi (cf. Mulckhuyse and Theeuwes, 2010). Instead, the fact that capture could be observed without subsequent IOR would be equally well in line with different origins of capture and IOR, for instance, an origin of capture in the early visual cortex (cf. Zhaoping, 2008) and an origin of IOR in posterior parietal cortex (cf. Toffanin et al., 2011).

Unexpectedly and in contrast to Mulckhuyse et al. (2007), our participants were able to objectively discriminate between the cues as indicated by significant $d^{\prime}$ values. This was the case even in conditions that were very similar in all important respects to the protocol of Mulckhuyse et al. (2007; present Exp. 4). Maybe some characteristic such as the exact shape of the cues (which were rings in our study but disks in Mulckhuyse et al., 2007) has led to a lower visibility in Mulckhuyse et al. (2007) study but our results are well in accordance with the known sensitivity of the visual system for temporal asynchronies between unconsciously cued and uncued stimuli (cf. Scharlau and Ansorge, 2003).

Note also that the currently found average ability of the participants to objectively discriminate between the cues does not cast doubts on our conclusion that we measured an unconscious capture effect. This is so because first, in Experiment 1, we showed 
very similar capture effects in objectively unconscious and conscious cueing conditions, that is, for participants that performed on chance level when discriminating the cues, a capture effect in the short SOA and no IOR in the long SOA was found, too. In addition, due to flicker fusion all of our participants failed to see the cues and therefore claimed to at least subjectively have remained unaware of the cues. All participants, therefore, passed a subjective criterion of unconscious cueing during target detection and this alone allows the conclusion that cueing was brought about unconsciously (Merikle et al., 2001). Crucially, the participants' inability of a strategic use of the anti-predictive unconscious cues clearly showed that the subliminal cues used in the present study were not consciously perceived (Exp. 5).

\section{CONCLUSION}

IOR can be found with visible and top-down matching cues (cf. Gibson and Amelio, 2000) and IOR is unreliable with unconscious exogenous capture (see our experiments above). Together, these observations are in line with a double dissociation between IOR on the one hand and unconscious exogenous capture on the other. The results are also suggestive of a decisive role of task sets

\section{REFERENCES}

Ansorge, U. (2003). Asymmetric influences of temporally vs. nasally presented masked visual information: evidence for collicular contributions to nonconscious priming effects. Brain Cogn. 51, 317-325.

Ansorge, U., Fuchs, I., Khalid, S., and Kunde, W. (2011). No conflict control in the absence of awareness. Psychol. Res. 75, 351-365.

Ansorge, U., Horstmann, G., and Worschech, F. (2010). Attentional capture by masked color singletons. Vision Res. 50, 2015-2027.

Ansorge, U., Kiss, M., and Eimer, M. (2009). Goal-driven attentional capture by invisible colors: evidence from event-related potentials. Psychon. Bull. Rev. 16, 648-653.

Ansorge, U., Kiss, M., Worschech, F., and Eimer, M. (2011). The initial stage of visual selection is controlled by top-down task set: new ERP evidence. Atten. Percept. Psychophys. 73, 113-122.

Baars, B. J. (2002). The conscious access hypothesis: origins and recent evidence. Trends Cogn. Sci. 6, 47-52.

Botvinick, M. M., Cohen, J. D., and Carter, C. S. (2004). Conflict monitoring and anterior cingulate cortex: an update. Trends Cogn. Sci. 8, 539-546.

Dorris, M. C., Klein, R. M., Everling, S., and Munoz, D. P. (2002). Contribution of the primate Superior Colliculus to inhibition of return. J. Cogn. Neurosci. 14, 1256-1263.
Folk, C. L., Remington, R. W., and Johnston, J. C. (1992). Involuntary covert orienting is contingent on attentional control settings. J. Exp. Psychol. Hum. Percept. Perform. 18, 1030-1044.

Fuchs, I., and Ansorge, U. (2012). Unconscious cueing via the superior colliculi: evidence from searching for onset and color targets. Brain Sci. 2, 33-60.

Gibson, B. S., and Amelio, J. (2000). Inhibition of return and attentional control settings. Percept. Psychophys. 62, 496-504.

Hu, F. K., and Samuel, A. G. (2011). Facilitation vs. inhibition in nonspatial attribute discrimination tasks. Atten. Percept. Psychophys. 73, 784-796.

Hunt, A. R., and Kingstone, A. (2003). Inhibition of return: dissociating attentional and oculomotor components. J. Exp. Psychol. Hum. Percept. Perform. 29, 1068-1074.

Itti, L., Koch, C., and Niebur, E. (1998). A model of saliency-based visual attention for rapid scene analysis. IEEE Trans. Pattern Anal. Mach. Intell. 20, 1254-1259.

Ivanoff, J., and Klein, R. M. (2003). Orienting of attention without awareness is affected by measurement-induced attentional control settings. J. Vis. 3, 32-40.

Kunde, W. (2003). Sequential modulations of stimulus-response correspondence effects depend on awareness of response conflict. Psychon. Bull. Rev. 10, 198-205.

and consciousness for inhibition in general and, thus, in good agreement with major theories of inhibitory executive functions (cf. Kunde, 2003; Botvinick et al., 2004; Ansorge, Fuchs et al., 2011). For instance, using conscious and unconscious cueing, IOR was stronger with conscious cues and sometimes selectively found with conscious cues (cf. Ivanoff and Klein, 2003). It is our opinion that the unreliable IOR effects of unconscious cues that have sometimes been reported in the literature (Mulckhuyse et al., 2007) could well be due to side factors unrelated to (I) the invisibility of the cues and (II) the nature of capture (i.e., whether capture was exogenous or not), such as the exact way in which the cue visibility has been reduced (cf. Ivanoff and Klein, 2003) or even the exact sample of participants (cf. Mulckhuyse et al., 2007). The absence of IOR after unconscious exogenous cueing in the present study certainly refutes Mulckhuyse and Theeuwe's (2010) assumption that IOR would be a hallmark of unconscious exogenous capture.

\section{ACKNOWLEDGMENTS}

Thanks to Alexander Kudrna, Isabell Möckel, Mira Seitzer, and Käthe Siuda for their valuable help with the data collection.

Lau, H. C., and Passingham, R. E. (2007). Unconscious activation of the cognitive control system in the human prefrontal cortex. $J$. Neurosci. 27, 5805-5811.

Lupiánez, J. (2010). "Inhibition of return," in Attention and Time, eds A. C. Nobre and J. T. Coull, (Oxford: Oxford University Press), 17-34.

Mack, A., and Rock, I. (1998). Inattentional Blindness. Cambridge, MA: The MIT Press.

Mayer, A. R., Seidenberg, M., Dorflinger, J. M., and Rao, S. M. (2004). An event-related fMRI study of exogenous orienting: supporting evidence for the cortical basis of inhibition of return? J. Cogn. Neurosci. 16, 1262-1271.

McCormick, P. A. (1997). Orienting attention without awareness. J. Exp. Psychol. Hum. Percept. Perform. 23, 168-180.

Merikle, P. M., Smilek, D., and Eastwood, J. D. (2001). Perception without awareness: perspectives from cognitive psychology. Cognition 79, 115-134.

Mulckhuyse, M., Talsma, D., and Theeuwes, J. (2007). Grabbing attention without knowing: automatic capture of attention by subliminal spatial cues. Vis. Cogn. 15, 779-788.

Mulckhuyse, M., and Theeuwes, J. (2010). Unconscious attentional orienting to exogenous cues: a review of the literature. Acta Psychol. (Amst) 143, 199-209.
Pratt, J., Sekuler, A., and McAuliffe, J. (2001). The role of attentional set on attentional cueing and inhibition of return. Vis. Cogn. 8, 33-46.

Prinzmetal, W., Taylor, J. A., Myers, L. B., and Nguyen-Espino, J. (2011). Contingent capture and inhibition of return: a comparison of mechanisms. Exp. Brain Res. 214, 47-60.

Rafal, R., Henik, R., and Smith, J. (1991). Extrageniculate contributions to reflex visual orienting in normal humans - a temporal hemifield advantage. J. Cogn. Neurosci. 3, 322-328.

Scharlau, I., and Ansorge, U. (2003). Direct parameter specification of an attention shift: evidence from perceptual latency priming. Vision Res. 43, 1351-1363.

Scharlau, I., Ansorge, U., and Horstmann, G. (2006). Latency facilitation in temporal-order judgments: time course of facilitation as a function of judgment type. Acta Psychol. (Amst) 122, 129-159.

Simons, D. J. (2000). Attentional capture and inattentional blindness. Trends Cogn. Sci. 4, 147-155.

Steinman, B. A., Steinman, S. B., and Lehmkuhle, S. (1997). Transient visual attention is dominated by the magnocellular stream. Vision Res. 37, 17-23.

Tassinari, G., and Berlucchi, G. (1993). Sensory and attentional components of slowing of manual reaction time to non-fixated visual targets by ipsilateral primes. Vision Res. 33, 1525-1534. 
Taylor, T. L., and Klein, R. M. (1998). On the cause and effects of inhibition of return. Psychon. Bull. Rev. 5, 625-643.

Toffanin, P., De Jong, R., and Johnson, A. (2011). The P4pc: an electrophysiological marker of attentional disengagement? Int. J. Psychophysiol. 81, 72-81.
Zhaoping, L. (2008). Attention capture by eye of origin singletons even without awareness - A hallmark of a bottom-up saliency map in the primary visual cortex. J. Vis. 8, $1-18$.

Conflict of Interest Statement: The authors declare that the research was conducted in the absence of any commercial or financial relationships that could be construed as a potential conflict of interest.

Received: 14 September 2011; accepted: 09 February 2012; published online: 24 February 2012.

Citation: Fuchs I and Ansorge U (2012) Inhibition of return is no hallmark of exogenous capture by unconscious cues. Front. Hum. Neurosci. 6:30. doi: 10.3389/fnhum.2012.00030

Copyright (c) 2012 Fuchs and Ansorge. This is an open-access article distributed under the terms of the Creative Commons Attribution Non Commercial License, which permits non-commercial use, distribution, and reproduction in other forums, provided the original authors and source are credited. 